
\title{
Reseacch Soute \\ Comparison of Short-Term Efficacy of Total Knee Arthroplasty Versus Arthroscopy in Chinese Patients with Knee Osteoarthritis
}

Xiaolin Jia ( $\nabla$ xiaolinjia20200420@163.com )

Chongqing General Hospital https://orcid.org/0000-0001-6702-9607

\section{San Cai}

Chongqing Public Health Medical Center

Wei Hu

Chongqing General Hospital

Qiang Gan

Chongqing General Hospital

Mingquan Zhou

Chongqing General Hospital

\section{Research}

Keywords: knee osteoarthritis, total knee arthroplasty, knee function

Posted Date: August 14th, 2020

DOl: https://doi.org/10.21203/rs.3.rs-56080/v1

License: (c) (1) This work is licensed under a Creative Commons Attribution 4.0 International License. Read Full License 


\section{Abstract}

Background: The purpose of this study was to compare the improvement of knee function in patients with knee osteoarthritis who underwent total knee arthroplasty and arthroscopy in China, and to provide a scientific basis for the application of clinical total knee arthroplasty in knee osteoarthritis.

Methods: A total of 160 patients with knee osteoarthritis who were admitted to Chinese hospital from January 2017 to December 2018 were studied. They were divided into experimental group and control group according to their willingness of treatment. The control group was treated with arthroscopy and the experimental group was treated with total knee arthroplasty. All patients were followed for a period of 6 months. The knee joint function score (HSS), visual analog scale (VAS), and anxiety self-assessment scale (SAS) scores before and after surgery were compared between the two groups.

Results: The proportion of "excellent or good" in the efficacy of the experimental group (91.25\%) was higher than that of the control group $(72.50 \%)$, and the difference was statistically significant $\left(\chi^{2}=9.476\right.$, $P<0.05)$. The HSS score of the experimental group was higher than that of the control group $(P<0.05)$, while the VAS and SAS scores were lower than those of the control group $(P<0.05)$. The scores of various SF-36 scales in the experimental group were higher than those in the control group after operation $(P<0.05)$.

Conclusions: Total knee arthroplasty was considered effective in treating patients with knee osteoarthritis that meet the indications, and is beneficial to improve knee function and reduce pain in patients. The surgical treatment also reduced the level of anxiety and effectively improve the quality of life of patients. Further investigation of its clinical application on treatment of knee osteoarthritis is warranted.

\section{Introduction}

Knee osteoarthritis is a condition based on degenerative pathological changes [1,2], it is more common in middle-aged and elderly people, and its symptoms are mostly red and swollen pain in the knee, pain in going up and down stairs, and sore knee discomfort when sitting and standing [3]. There will also be patients with swelling, popping, effusion, etc [4]. If not treated in time, it will cause joint deformities or disability [5]. Knee joints often also suffer from joint diseases such as knee synovitis, ligament damage, meniscus injury, knee free bodies, popliteal cysts, patellar softening, goose foot bursitis, and knee varus [6].

Knee osteoarthritis is one of the more common clinical diseases in orthopedics [7, 8]. It mainly refers to chronic bone and joint diseases caused by knee cartilage degeneration and bone hyperplasia, which are more common in the elderly $[9,10]$. Most patients with knee arthritis have milder initial symptoms and will gradually worsen if left untreated. The main symptoms are sore knees, swelling of the knee joints, and knee popping. Knee joint stiffness and chills are also one of the symptoms of knee arthritis [11]. It is mainly aggravated by stiffness, fatigue, cold or minor trauma, and severe cases will have limited mobility [12]. The occurrence of knee arthritis is usually caused by degenerative lesions of the knee joint, trauma, 
overwork and other factors. Knee arthritis is more common in the elderly and is the main cause of leg pain in the elderly [13]. In addition, overweight, incorrect walking posture, prolonged squatting, cold and cold knee joints are also the causes of knee arthritis.

So far, surgical treatment has been widely used in clinical osteoarthritis $[3,14]$. This treatment method can not only significantly improve the knee joint function of patients, but also help reduce the knee pain of patients, and the prognosis is good $[15,16]$. And domestic research reports have confirmed that the use of total knee arthroplasty for knee osteoarthritis patients can significantly improve the function and mobility of the knee joint. At the same time, it is conducive to reducing the occurrence of a series of complications such as infection, dislocation, deep vein thrombosis, and vascular injury, and it is helpful to promote the early recovery of patients. In view of this, this study explores knee function scores (HSS), visual analogue scoring (VAS), and self-rating anxiety scale (SAS), in order to provide theoretical basis for the application of clinical total knee arthroplasty in knee osteoarthritis.

\section{Methods}

\section{Collection of general information}

A total of 160 patients with knee osteoarthritis who were treated in Chinese hospital from January 2017 to December 2018 were selected as the research subjects. They were divided into experimental group and control group according to their willingness of treatment. There were 80 cases in each group. The study protocol was approved by the hospital medical ethics committee and written informed consent was obtained from all patients before treatment. Diagnostic criteria for knee osteoarthritis in need of surgery was based on the 2007 edition of Guidelines for the Diagnosis and Treatment of Osteoarthritis [17]: (i) there was obvious repeated knee pain within 1 month before admission; (ii) X-ray examination revealed that the joint space was narrow, the subchondral bone was hardened or cystic, and osteophytes formed at the joint edges; (iii) the joint fluid is clear and the white blood cell (WBC) level is lower than 2000 cells/ml; (iv) patients at age more than 40 years old; (v) morning stiffness time $\leq 30 \mathrm{~min}$; and (vi) bone rubbing feeling during activities. Those who met the above criteria (i) and (ii), or (i), (iii), (v) and (vi), or(i), (iv), (v) and (vi) can be diagnosed as osteoarthritis.

\section{Inclusion and exclusion criteria}

Inclusion criteria were: (i) all patients met the above diagnostic criteria; (ii) age of more than or equal to 45 years old; (iii) those who did not receive any related treatment before study admission; and (iv) patients with complete clinical and medical records. Exclusion criteria were: (i) patients with severe dysfunction of heart, liver, kidney and other organs; (ii) persons with severe infectious disease or immune disease; (iii) patients with rheumatoid arthritis, joint tuberculosis, gout and bone tumor disease; (iv) persons with neurological diseases or communication difficulties; and (v) person who is participating in other studies.

\section{Surgical methods}


Patients treated with knee arthroscopic surgery were categorized as control group. Under epidural block anesthesia, patient was on supine position and the knee-eye approach was performed. During arthroscopy, the joints were lavaged and the proliferated synovial membrane, cuff, cartilage and debris were removed. The meniscus was trimmed, the cartilage was polished, and the free body was removed according to surgeon's judgment. Then the joints are rinsed, and the incision is sutured after completion of the aspiration, and the bandage is compressed. After surgery, patient's joint was compressed with ice for 12 hours and the drainage tube was removed 48 hours postoperatively. Patients were treated with conventional antibiotics and anticoagulants to prevent infection and thrombosis after the arthroscopic knee surgery.

Patients treated with total knee arthroplasty were categorized as experimental group. All patients were also under epidural block anesthesia for surgery. Incision was made at the anterior median part of the knee joint and the joint capsule was then incised in the superficial layer to the patella to determine the joint disease. Osteophytes, meniscus, cruciate ligament and submental fat pad were excised, and the soft tissue around the knee joint was then removed according to the specific conditions of the patient, and tibia and femur osteotomy were performed at the same time. The distal femoral joint was kept valgus of 5 to 7 degrees and externally rotated 3 degrees. After completion of prosthesis test, the corresponding knee prosthesis was implanted with the use of bone cement. After the total knee replacement operation, a negative pressure drainage tube was placed in the wound, and the incision area was closed with sutures. After the ice was applied for 48 to 72 hours, the drainage tube was removed and patients were treated with antibiotics and anticoagulants. All patients were followed for a period of 6 months.

\section{Observation indicators}

Changes in HSS, VAS and SAS scores, treatment efficacy, and quality of life of patients were compared between two groups before and after surgery. Among them, HSS is mainly used to evaluate the functions of patients' knee joints, mainly including joint stability, joint muscle strength, range of motion, joint function, joint pain, and degree of joint flexion deformity [18]. The total score is 100 points. A higher score indicates better knee function. VAS is mainly used to assess the pain intensity of patients, with a total score of 0 to 10 points, and the higher the score, the more severe the patient's pain [10]. SAS is mainly used to assess the psychological anxiety of patients. It contains 20 items, of which SAS scores 1 to 4 points. The higher the score, the more severe the patient's anxiety [19]. The curative effect is mainly judged based on the HSS score: an HSS score of $\geq 84$ is excellent; an HSS score of $80-83$ is good; an HSS score of 60-69 is acceptable; an HSS score of $<60$ is poor. Excellent rate $=$ (excellent number + good number) / total number of cases $\times 100 \%$. The Short Form-36 (SF-36) scale was used to evaluate and analyze the quality of life of patients, including eight aspects such as physiological function, physical function, physical pain, general health, social function, vitality, emotional function and mental health.

There are 100 points for each aspect. A higher score indicates a better quality of life.

\section{Statistical Analysis}


Data analysis was performed using SPSS 21.0 statistical software. Measurement data were expressed as mean \pm standard deviation $(\mathrm{M} \pm \mathrm{SD})$, and comparison was performed using t-test. Counting data were expressed by examples and percentage, and comparison was made by $\chi^{2}$ test. P-value of less than 0.05 was considered statistically significant.

\section{Results}

\section{Analysis of general demographic characteristics and surgical efficacy of the two groups of respondents}

The control group included 45 males and 35 females, with an average age of $59.31 \pm 5.49$ years and a course of disease of $4.41 \pm 0.71$ years. The subjects in the experimental group were 41 males and 39 females, with an average age of $58.77 \pm 6.65$ years and a course of disease of $4.42 \pm 0.76$ years. There were no significant differences in gender, age, and duration of disease between the two groups of participants $(P>0.05)$.

In the control group, the curative effect was excellent in 28 cases, good in 30 cases, fair in 15 cases, and poor in 7 cases, while the participants in the experimental group had 37 cases with excellent surgical outcomes, 36 cases with good outcomes, 6 cases with fair outcomes, and 1 case with poor outcome. There were a total of 73 (91.25\%) patients achieving "excellent or good" outcomes after treatment with total knee arthroplasty, which was higher than that observed in $58(72.5 \%)$ patients treated with knee arthroscopic surgery with necessary meniscal resection or other surgical procedures as indicated, and the difference was statistically significant $(x 2=9.476, P<0.05)$. The results of statistical analysis are shown in Table 1.

\section{HSS, VAS, and SAS scores before and after surgery in the two groups}

There was no significant difference in the HSS, VAS, and SAS scores between the control group and the experimental group before surgery $(P>0.05)$. After surgery, the HSS score of the experimental group was statistically higher than that of the control group, while the VAS and SAS scores were statistically lower than those of the control group $(P<0.001)$. Results are shown in Table 2.

\section{SF-36 scores before and after surgery in the two groups}

There was no significant difference in the scores of SF-36 before surgery between the two groups ( $P>0.05)$. The scores of various SF-36 sub-scales after the operation were also compared. Each score of the SF-36 scale of patients in the experimental group was statistically higher than those in the control group $(P<0.001)$ which represents a better quality of life was observed in patients treated with the total knee arthroplasty. The results of statistical analysis are shown in Table 3 and 4.

\section{Discussion}


Knee osteoarthritis is a degenerative disease of the knee cartilage caused by multiple factors and secondary bone hyperplasia $[16,20]$. The main clinical manifestations are knee pain, limited mobility, and deformity. Some patients are accompanied by joint swelling and stiffness, which seriously affects their quality of life [21]. Knee osteoarthritis is one of multiple chronic joint diseases with a high incidence. The incidence of knee osteoarthritis among people over 40 years old in China ranges from $10 \%$ to $17 \%$, while the incidence of people over 60 years old is $50 \%$, and the incidence of people over 75 years of age is as high as $80 \%$ [22]. Total knee arthroplasty used to be the surgical treatment for ineffective knee joint disease. It helps improve joint functions, reduce joint pain, and minimize the adverse impact of the disease on patients' daily living. However, the surgery may not be always successful due to various reasons [23, 24], for example, the postoperative pain may become more severe. Combining the effects of joint swelling and intra-articular hemorrhage, patients may be difficult to recover after surgery physically, and in turn seriously affect the patient's psychological well-being.

Nevertheless, the results of this study indicated that the ratio of "excellent and good" surgical outcomes in the experimental group was $91.5 \%$, which was higher than that of $72.5 \%$ in the control group. This showed that total knee arthroplasty achieved a good treatment outcomes in patients with knee osteoarthritis and could effectively improve the knee function of patients. From our clinical experience, this treatment method can effectively clear the soft tissue around the knee joint by removing the submental fat pad, meniscus, cruciate ligament and osteophytes, and further avoids the deviation of the tibial prosthesis and the loosening of the prosthesis. At the same time, the stability and mobility of the knee joint of the patient are enhanced, wear of the inner pad between the joints is delayed, and the purpose of improving the knee function of the patient can then be finally achieved. Total knee arthroplasty is the main treatment method for patients with knee joint disease who are ineffective after various conservative treatments. Among them, degenerative osteoarthrosis of the knee joint is the most common joint diseases [25]. After patients undergo total knee arthroplasty, joint pain can usually be significantly improved, and local deformities can be effectively corrected. This will improve the knee function of the patient and keep the knee joint stable for a long time.

According to this study, the HSS score of the experimental group was higher than that of the control group, and the VAS and SAS scores were lower than those of the control group. The results suggested that treatment of total knee arthroplasty in patients with knee osteoarthritis will help improve their knee functions, and reduce their levels of pain and anxiety. Total knee arthroplasty is a simple operation which timely corrects knee joint deformity of the patient. This treatment is beneficial to maintain the balance of the soft tissue in the knee joint, thereby ensuring the stability of the knee joint. It also helps avoid the pain and other series of symptoms caused by the decline of joint performance, and further effectively alleviate the negative emotions such as anxiety and fear caused by pain.

The study also demonstrated that the scores of various SF-36 scales in the experimental group were higher than those in the control group. The results suggested that total knee arthroplasty can improve the quality of life of patients with knee osteoarthritis. The main reason may be related to the effective improvement of knee joint function, reduction of pain and anxiety, and promotion of the recovery of 
patients' physical and mental health [26]. When performing total knee arthroplasty on patients with knee osteoarthritis, it is necessary to improve the condition analysis, clarify the type of medial soft tissue, and the main treatment goals are to correct knee dislocation and improve tissue loosening.

\section{Conclusions}

In summary, total knee arthroplasty can be considered as one of the treatment choices for patients with knee osteoarthritis. The treatment significantly improves knee function, physical and psychological wellbeing of patients, and thus beneficial to improving the quality of life of patients. Further investigation on the long-term outcomes and impact may unveil the full potential of this surgical treatment for knee osteoarthritis.

\section{List Of Abbreviations}

HSS: knee function scores

VAS: visual analogue scoring

SAS: self-rating anxiety scale

WBC: white blood cell

SF-36: Short Form-36

$\mathrm{M} \pm$ SD: mean \pm standard deviation

\section{Declarations}

\section{Ethics approval and consent to participate}

The study was approved by the Ethics Committee of Chongqing General Hospital. Patients who participated in this research, signed the informed consent and had complete clinical data.

\section{Consent for publication}

Not applicable.

\section{Availability of data and material}

The datasets used or analysed during the current study are available from the corresponding author on reasonable request.

\section{Competing interests}

The Authors declare that they have no competing interests. 


\section{Funding}

This work was supported by Chongqing Medical Scientific Research Program [grant number 2016HBRC005].

\section{Authors' contributions}

$\mathrm{XJ}$ designed this study and helped with statistical analysis. SC and WH collected and analyzed general data of patients. QG and MZ were responsible for CT and MRI result analysis. All authors read and approved the final manuscript.

\section{Acknowledgements}

Not applicable.

\section{References}

1. Zafar AQ, Zamani R and Akrami M. The effectiveness of foot orthoses in the treatment of medial knee osteoarthritis: A systematic review. Gait Posture. 2020;76:238-251.

2. Wada O, Kurita N, Kamitani T, Mizuno K. Implications of evaluating leg muscle mass and fat mass separately for quadriceps strength in knee osteoarthritis: the SPSS-OK study. Clin Rheumato 2020;39:1655-1661.

3. Fantini Pagani C, Funken J, Heinrich K, Ellermann A, Schmidt-Wiethoff R, Potthast W. Predicting the knee adduction moment after high tibial osteotomy in patients with medial knee osteoarthritis using dynamic simulations. Knee. 2020;27:61-70.

4. Maclntyre E, Sigerseth M, Pulling BW, Newport R, Stanton TR. The effect of knee resizing illusions on pain and swelling in symptomatic knee osteoarthritis: a case report. Pain Rep. 2019;4:e795.

5. Parisi S, Ditto MC, Priora M, Borrelli R, Lagana A, Peroni CL, et al. Ultrasound-guided intra-articular injection: efficacy of hyaluronic acid compared to glucocorticoid in the treatment of knee osteoarthritis. Minerva Med. 2019;110:515-523.

6. Goodman SM, Mehta BY, Mandl LA, Szymonifka JD, Finik J, Figgie MP, et al. Validation of the Hip Disability and Osteoarthritis Outcome Score and Knee Injury and Osteoarthritis Outcome Score Pain and Function Subscales for Use in Total Hip Replacement and Total Knee Replacement Clinical Trials. J Arthroplasty. 2020;35:1200-1207.

7. Nayak M, Kumar V, Kanojiya G, Mellon S, Srivastava DN, Pandit H, et al. A radiographic analysis of alignment in 966 lower extremities with knee pain and its association with osteoarthritis in Indian population. J Orthop. 2019;20:207-212.

8. Tiulpin A, Klein S, Bierma-Zeinstra SMA, Thevenot J, Rahtu E, Meurs JV, et al. Multimodal Machine Learning-based Knee Osteoarthritis Progression Prediction from Plain Radiographs and Clinical Data. Sci Rep. 2019;9:20038. 
9. Garg Y, Singh J, Sohal HS, Gore R, Kumar A. Comparison of Clinical Effectiveness and Safety of Newer Nonsteroidal Anti-inflammatory Drugs in Patients of Osteoarthritis of Knee Joint: A Randomized, Prospective, Open-label Parallel-group Study. Indian J Pharmacol. 2017;49:383-389.

10. Fu J, Shang HC, Wang LY, Zhao C, Cui J, Wang YP. Crossbow needle therapy of the Miao ethnic minority group for knee osteoarthritis: study protocol for a randomized controlled trial. Trials. 2018;19:338.

11. Ackerman IN, Cavka B, Lippa J, Bucknill A. The feasibility of implementing the ICHOM Standard Set for Hip and Knee Osteoarthritis: a mixed-methods evaluation in public and private hospital settings. J Patient Rep Outcomes. 2017;2:32.

12. Srivastava S, Chaudhary JA, Girandola RN. Effect Of E-OA-07 On Improving Joint Health And Mobility In Individuals With Knee Osteoarthritis: A Randomized, Double-Blind, Placebo-Controlled, Parallel Group Study. J Pain Res. 2019;12:3365-3379.

13. Matada MS, Holi MS, Raman R, Jayaramu Suvarna ST. Visualization of Cartilage from Knee Joint Magnetic Resonance Images and Quantitative Assessment to Study the Effect of Age, Gender and Body Mass Index (BMI) in Progressive Osteoarthritis (OA). Curr Med Imaging Rev. 2019;15:565-572.

14. Sethi G, Aljawadi A, Elmajee M, Pillai A. Determination of the postoperative limb alignment following a high tibial osteotomy in patients with uni-compartmental knee osteoarthritis, review article. J Orthop. 2020;18:53-57.

15. Migliore A, Gigliucci G, Alekseeva L, Avasthi S, Bannuru RR, Chevalier X, et al. Treat-to-target strategy for knee osteoarthritis. International technical expert panel consensus and good clinical practice statements. Ther Adv Musculoskelet Dis. 2019;11:1759720X19893800.

16. Riddle DL. Prevalence of similar or worse symptom and osteoarthritis severity of index and contralateral knees prior to knee arthroplasty: A cross-sectional multicenter cohort study. Knee. 2020;27:485-492.

17. Nie Y, Wang H, Xu B, Zhou Z, Shen B, Pei F. The Relationship between Knee Adduction Moment and Knee Osteoarthritis Symptoms according to Static Alignment and Pelvic Drop. Biomed Res Int. 2019;2019:7603249.

18. Qiu GX. Guidelines for the diagnosis and treatment of osteoarthritis (2007 edition). Chin Joint Surg. 2007;1:281-285.

19. Burn E, Liddle AD, Hamilton TW, Judge A, Pandit HG, Murray DW, et al. Cost-effectiveness of unicompartmental compared with total knee replacement: a population-based study using data from the National Joint Registry for England and Wales. BMJ open. 2018;8:e20977.

20. Pinkaew D, Kiattisin K, Wonglangka K, Awoot P. Phonophoresis of Phyllanthus amarus nanoparticle gel improves functional capacity in individuals with knee osteoarthritis: A randomized controlled trial. J Bodyw Mov Ther 2020;24: 15-18.

21. Kumar H, Pal CP, Sharma YK, Kumar S, Uppal A. Epidemiology of knee osteoarthritis using Kellgren and Lawrence scale in Indian population. J Clin Orthop Trauma. 2020;11:S125-S129. 
22. Lu W, Zhou S, Xue L, Dai B, Qiao L, Chen D, et al. High preoperative serum leptin level is an independent risk factor for deep vein thrombosis after total knee arthroplasty in osteoarthritis patients: A prospective and cross-sectional study. Medicine. 2018;97:e10884.

23. Eloa MM, Carla FD, Danielle SM, Danubia CS, Cintia RS, Laisa LP, et al. Whole body vibration and auriculotherapy improve handgrip strength in individuals with knee osteoarthritis. J Tradit Chin Med. 2019;39:707-715.

24. Haydel A, Guilbeau S, Roubion R, Leonardi C, Bronstone A, Dasa V. Achieving Validated Thresholds for Clinically Meaningful Change on the Knee Injury and Osteoarthritis Outcome Score After Total Knee Arthroplasty: Findings From a University-based Orthopaedic Tertiary Care Safety Net Practice. J Am Acad Orthop Surg Glob Res Rev. 2019;3:e00142.

25. Ariani A, Manara M, Fioravanti A, lannone F, Salaffi F, Ughi N, et al. The Italian Society for Rheumatology clinical practice guidelines for the diagnosis and management of knee, hip and hand osteoarthritis. Reumatismo. 2019;71:5-21.

26. Sundaram K, Klare CM, Moschetti WE. Proximal tibiofibular osteoarthritis presenting as pain after total knee arthroplasty treated successfully with fusion of the proximal tibial-fibular joint. Arthroplast today. 2018;4:139-142.

\section{Tables}

Table 1

Analysis of general demographic characteristics of survey respondents

\begin{tabular}{|lllll|}
\hline Variables & Control group $(\mathrm{n}=80)$ & Experimental group $(\mathrm{n}=80)$ & $x^{2}$ or $t$ & $P$ \\
\hline Age(years) & $59.31 \pm 5.49$ & $58.77 \pm 6.65$ & 0.558 & 0.578 \\
\hline Course of disease (years) & $4.41 \pm 0.71$ & $4.42 \pm 0.76$ & -0.092 & 0.927 \\
\hline Gender & & & & \\
\hline Male & 45 & 41 & 0.402 & 0.526 \\
\hline Female & 35 & 39 & & \\
\hline Surgical outcome & & & & \\
\hline Excellent & 28 & 37 & & \\
\hline Good & 30 & 36 & & \\
\hline Fair & 15 & 6 & & \\
\hline Poor & 7 & 1 & & \\
\hline
\end{tabular}


Table 2

HSS, VAS, and SAS scores before and after surgery in the two groups

\begin{tabular}{|clllll|}
\hline \multicolumn{2}{|l}{ Variables } & Control group & Experimental group & $t$ & $P$ \\
\hline \multirow{2}{*}{ HSS } & Before surgery & $41.96 \pm 6.03$ & $41.88 \pm 5.86$ & 0.085 & 0.932 \\
\cline { 2 - 6 } & After surgery & $70.75 \pm 6.00$ & $84.92 \pm 7.24$ & -13.488 & $<0.001$ \\
\hline \multirow{2}{*}{ VAS } & Before surgery & $5.66 \pm 0.89$ & $5.56 \pm 0.76$ & 0.717 & 0.474 \\
\cline { 2 - 6 } & After surgery & $4.16 \pm 0.73$ & $2.22 \pm 0.58$ & 18.676 & $<0.001$ \\
\hline \multirow{2}{*}{ SAS } & Before surgery & $42.81 \pm 9.74$ & $41.18 \pm 8.59$ & 1.12 & 0.265 \\
\cline { 2 - 6 } & After surgery & $30.03 \pm 6.62$ & $19.54 \pm 6.39$ & 10.199 & $<0.001$ \\
\hline
\end{tabular}

Table 3

Comparison of the scores of the SF-36 scale before surgery between the two groups of respondents

\begin{tabular}{|lllll|}
\hline Variables & Control group & Experimental group & $t$ & $P$ \\
\hline Physiological function & $27.43 \pm 5.55$ & $26.70 \pm 5.74$ & 0.82 & 0.413 \\
\hline Physical function & $30.63 \pm 6.32$ & $31.28 \pm 6.80$ & -0.626 & 0.532 \\
\hline Physical pain & $33.01 \pm 7.24$ & $33.12 \pm 7.69$ & -0.099 & 0.921 \\
\hline General health & $28.91 \pm 8.35$ & $29.19 \pm 8.32$ & -0.207 & 0.836 \\
\hline Social function & $31.54 \pm 8.81$ & $29.41 \pm 9.93$ & 1.438 & 0.152 \\
\hline Vitality & $42.93 \pm 9.19$ & $43.82 \pm 7.16$ & -0.678 & 0.499 \\
\hline Emotional function & $30.33 \pm 9.35$ & $29.57 \pm 9.43$ & 0.511 & 0.61 \\
\hline Mental health & $35.81 \pm 8.41$ & $35.35 \pm 10.31$ & 0.309 & 0.758 \\
\hline
\end{tabular}


Table 4

Comparison of the scores of the SF-36 scale after surgery between the two groups of respondents

\begin{tabular}{|lllll|}
\hline Variables & Control group & Experimental group & $t$ & $P$ \\
\hline Physiological function & $61.56 \pm 5.70$ & $69.67 \pm 6.89$ & -8.108 & $<0.001$ \\
\hline Physical function & $61.67 \pm 7.11$ & $71.24 \pm 6.62$ & -8.823 & $<0.001$ \\
\hline Physical pain & $65.04 \pm 7.86$ & $75.65 \pm 8.93$ & -7.974 & $<0.001$ \\
\hline General health & $63.42 \pm 7.2$ & $75.84 \pm 8.42$ & -10.026 & $<0.001$ \\
\hline Social function & $63.15 \pm 10.17$ & $79.23 \pm 9.53$ & -10.319 & $<0.001$ \\
\hline Vitality & $71.91 \pm 9.58$ & $80.56 \pm 8.69$ & -5.988 & $<0.001$ \\
\hline Emotional function & $63.59 \pm 7.97$ & $74.5 \pm 8.36$ & -8.454 & $<0.001$ \\
\hline Mental health & $62.4 \pm 6.68$ & $72.05 \pm 7.58$ & -8.535 & $<0.001$ \\
\hline
\end{tabular}

